\title{
Social Determinants of Malaria Treatment Seeking Time by Mothers for Children (0-5 Years) in South Eastern Nigeria
}

\author{
Chukwuocha UM ${ }^{1,3 *}$, Okpanma $\mathrm{AC}^{1,2}$, Chukwuocha $\mathrm{AN}^{1,3}$ and Nwakwuo $\mathrm{GC}^{1,3}$
}

${ }^{1}$ Department of Public Health Technology, Federal University of Technology, Owerri, Imo State Nigeria

${ }^{2}$ School of Nursing, Ministry of Health, Owerri, Imo State Nigeria

${ }^{3}$ Community Initiative for Infectious Disease Control (CIIDC), Nigeria

\begin{abstract}
This study aimed at investigating the influence of social characteristics of mothers on time taken to seek treatment for their children suspected of having malaria in four rural communities in south-eastern Nigeria. Data were from 738 consenting mothers within the child bearing age (15-49 years) using structured pre-tested questionnaires. Results showed that (22\%) sought treatment within 24 hours for their children (0-5years). More than half $(51.5 \%)$ of those who sought treatment for their children after 24 hours, did so (delayed) because they had to watch their children for some days, while $21.4 \%$ were due to financial difficulties. The age, parity, marital status/type of marriage and educational attainment of the mothers including family social-economic status were found to be statistically related to delay in seeking prompt and appropriate malaria treatment. Wrong first line treatment choices by the respondents also contributed to their delay. Suggestions were made based on these findings on how to eliminate delay in seeking and commencing early and appropriate treatment for childhood ( $0-5$ years) malaria..
\end{abstract}

\section{Keywords: Malaria; Artemesinin; Rural communities}

\section{Introduction}

Malaria has continued to be a major public health problem in the World today despite concerted efforts aimed at its control and elimination. The organism that causes the illness (plasmodium) spends parts of its life cycle in the mosquito and the other in man [1].

Infected persons usually experience fever (increased body temperature), joint pains and aches, headache, malaise (general body weakness) etc. Infection by malaria can be classified by its severity into uncomplicated and severe malaria. The diagnosis of malaria is mostly through its signs and symptoms, although the diagnosis is confirmed through a microscopic examination of blood film in the laboratory. Malaria can also be diagnosed through "Rapid Diagnostic Test" (RDT) strip. This later method is easier, quicker and can be used in the rural communities where there are no laboratories. According to the World Health Organization (WHO) treatment guidelines, the most effective treatment for uncomplicated malaria (which cause $98 \%$ of malaria cases in Nigeria) is Artemesinin based combination therapy (ACT). Malaria prevention is achieved by preventing mosquito bites through taming the environment to prevent the breeding of mosquito, use of insecticides to kill the vector, early recognition and treatment of victims to avoid further transmission and prevent complications.

Complications of malaria arise when malaria treatment is delayed or not properly managed. These complications include cerebral malaria, pulmonary oedema, kidney failure, liver failure, spleen rupture, anaemia, hypoglycaemia etc. [2].

According to Chan [3], 216 million cases of malaria occurred in $2010,91 \%$ in African region and $86 \%$ were children under five years of age. Furthermore, one out of five deaths of children under- five years in Africa is due to malaria. In Africa, an estimated 300-500 million cases of malaria occur each year resulting in approximately One million deaths. Among deaths occurring in Africa due to malaria, more than $90 \%$ are in under-five children as a result of brain damage [4]. United States Embassy in Nigeria observed that Malaria accounts for more cases and deaths in Nigeria than in any other country in the world [5]. It is a risk for $97 \%$ of Nigerias population; the remaining $3 \%$ of the population live in the malaria free highlands. And there are an estimated 100 million malaria cases with over 300,000 deaths per year. Therefore, it is a public health concern (in Nigeria) and is present throughout the country. It accounts for $60 \%$ of out-patient visits and $30 \%$ of hospitalizations among children under five years of age [6,7]. The disease is major cause of morbidity and mortality among children under five years of age in Nigeria. It is widely recognized that despite global, national and local initiatives and programmes, this situation is not improving quickly enough [8].

One of the main components of the World Health Organization current strategy centres on early recognition and prompt treatment. Early diagnosis, prompt and effective treatment has been recognized as the basis for the management of malaria and the key to reducing malaria morbidity and mortality including its transmission $[9,10]$.

Most the treatments for malaria and other febrile conditions in Nigeria are however through self-medication with anti-malaria bought over the counter (OTC) from untrained drug vendors [11].

But the concept of treatment seeking especially as regards to time taken from recognition of the illness to do so involves how people employ the healthcare systems in their respective socio-cultural, economic circumstances. All these define social position of health and provide better understanding of the disease process. The determinants of health care seeking therefore extend beyond health care and can be attributed to these social and economic variables which most health

*Corresponding author: Chukwuocha U.M, Department of Public Health Technology, Federal University of Technology, Owerri, Imo State Nigeria, Tel: +2348034712957; E-mail: : uchukwuocha@gmail.com

Received February 03, 2015; Accepted March 09, 2015; Published March 16 2015

Citation: Chukwuocha UM, Okpanma AC, Chukwuocha AN, Nwakwuo GC (2015) Social Determinants of Malaria Treatment Seeking Time by Mothers for Children (0-5 Years) in South Eastern Nigeria. J Trop Dis 2: 154. doi $10.4172 / 2329891 X .1000154$

Copyright: (c) 2013 Chukwuocha UM, et al. This is an open-access article distributed under the terms of the Creative Commons Attribution License, which permits unrestricted use, distribution, and reproduction in any medium, provided the original author and source are credited. 
Citation: Chukwuocha UM, Okpanma AC, Chukwuocha AN, Nwakwuo GC (2015) Social Determinants of Malaria Treatment Seeking Time by Mothers for Children (0-5 Years) in South Eastern Nigeria. J Trop Dis 2: 154. doi: 10.4172/2329891X.1000154

systems have not been able to link with the health of their populations [12].

A major problem that hinders effective treatment and management of malaria is delay in treatment seeking. According to Safer et al. [13], delay in treatment seeking has three stages thus: appraisal delay, illness delay and utilization delay.

Appraisal delay: This refers to the time taken by a patient to appraise a symptom as a sign of illness.

Illness delay: Is the time taken from deciding one is ill to deciding to seek professional medical care.

Utilization delay: This is the time from the decision to seek care until the patient goes to the clinic and uses its services.

Several factors affect the three stages of delay which range from disorder characteristics, subject (individual) characteristics to service characteristics [14]. The disorder characteristics include the aetiological agent of the illness, nature/stage of the illness (acute or chronic) expected benefits from treatment etc. The disorder characteristics also include all the socio-demographic data such as age, sex, marital status, ethnicity, education, occupation, finance etc. The service characteristics include accessibility, acceptability, quality, cost, type of service (traditional or orthodox) etc.

This study is therefore aimed at investigating the influence of mother's social characteristics on time taken to seek treatment for their children suspected of having malaria.

\section{Materials and Methods}

\section{Study area}

This cross sectional descriptive study was conducted from January to October 2013, in four rural communities in Ohaji/Egbema local government area of Imo State Nigeria. Imo State is one of the thirtysix states of the Federal Republic of Nigeria, found between latitude $5^{\circ} 10^{`}-5^{\circ} 51^{\prime}$ North of the Equator and longitude $6^{\circ} 35^{`}-7^{\circ} 28^{\prime}$ East of the Greenwich Meridian.

The oil rich Ohaji/Egbema local government area of Imo State has a population of 182,538 according to the National Population Commission in 2006, which is projected to be 227,568 in 2012 , according to the health department of the local government. The local government has a vast area of arable land (890 KM2) used for agriculture with some areas of tropical rain forest and mangrove swamps lining the western boundaries with Rivers State due to the presence of the Orashi River and its tributaries. Sequel to these climatic conditions, the state has palm and rubber plantations in Ohaji and a forest reserve in Egbema.

The people of Ohaji/Egbema local government area are predominantly farmers and fishermen who at times sleep in huts constructed in their farms in order to increase production by having more working hours and less distraction. At home the housing style involves the construction of kitchen and toilets some distance away from the main houses. Most of the houses do not have mosquito proof netting and proper drainage systems.

Environmental factors such as poor waste disposal - open dumping, poor drainage system, farms and gardens around the houses, plantain, banana and cocoyam suckers, community ponds etc. provide good breeding places for the multiplication of mosquitoes the vector of falciparum malaria .
The vector which is the female Anopheles gambiae, has the highest population density during the rainy season. During the dry season, people in this area are forced to spend reasonable time outside their houses at nights in bid to have cool air as some of the communities do not have electricity; even those who have suffer epileptic power supply. By this people are exposed to frequent mosquito bites and subsequent transmission of the disease all year round.

\section{Sampling and data collection}

A cross sectional descriptive design was employed for this study. The respondents included all women of child bearing age ( $15-49$ years) whether they currently have children below five years or not in the four selected communities. Women were used as the study population because 'mothers regardless of their socio-demographic characteristics make the first diagnosis of their child's febrile illness by defining and interpreting changes in their child's behavior and temperature' [15]. Women who had never had children for the first time were excluded from the study. About 738 mothers representing $5 \%$ of the total number of women of child bearing age $(14,758)$ in the four communities were selected through balloting. Informed oral consent was sought and obtained from these women.

Data was collected using structured pre-tested questionnaires administered by trained field assistants. The questionnaires were first pretested among a group of twenty women who attended Immunization services at the Health Centre located in each of the four selected communities. Some respondents who could not write or have time for completing the questionnaires were assisted. The questions were elicited in the local Igbo Language The word 'Malaria' was translated into native language as 'Akom' or 'Iba'.

\section{Data analysis}

The data gathered from the respondents were checked manually for completeness and then entered into Epi-Info version 6.04. Analysis was done using SPSS version 20.0 and Chi-Square.

\section{Results}

Table 1 shows the socio-demographic characteristics of the respondents. The most represented age group was $22-28$ years $(32.8 \%)$ with married/monogamous mothers making the greater portion of the respondents $350(47.4 \%)$. The most common occupation of the mothers is farming $252(34.1 \%)$.This is followed by trading which is 185 representing 25.1\%. Almost half of the respondents 336(45.5\%) had only primary education, followed by secondary education 259 representing (35.1\%). Those with Bachelor of Science degree and above were the least 119 , which represented $16.1 \%$. The least represented group was those without any formal education 24 (3.3\%). In monthly income distribution as in Table 1 the majority of the respondents 418 representing $56.6 \%$ were low income earners. This was followed by the middle income earners with $190(25.8 \%)$ and lastly the high income earners who were only 130 (17.6\%).

In this study $163(22 \%)$ respondents sought malaria treatment within 24 hours for children (0-5 years) (Not delayed) while 575(78\%) respondents did not seek malaria treatment within 24 hours for their children (Delayed).

\section{Reasons for not seeking malaria treatment within 24 Hours}

The 575 (78\%) respondents did not seek malaria treatment for their children within 24 hours. Their reasons are shown in Figure 1. More than half of them would rather watch the child for some days to be sure 
Citation: Chukwuocha UM, Okpanma AC, Chukwuocha AN, Nwakwuo GC (2015) Social Determinants of Malaria Treatment Seeking Time by Mothers for Children (0-5 Years) in South Eastern Nigeria. J Trop Dis 2: 154. doi: 10.4172/2329891X.1000154

\begin{tabular}{|c|c|c|}
\hline Variables & Frequency & Percentage \\
\hline \multicolumn{3}{|l|}{ Age } \\
\hline $15-21 y r s$ & 88 & 11.9 \\
\hline $22-28 y r s$ & 242 & 32.8 \\
\hline $29-35 y r s$ & 221 & 30.0 \\
\hline $36-42 y r s$ & 167 & 22.6 \\
\hline $43-49 y r s$ & 20 & 2.7 \\
\hline \multicolumn{3}{|l|}{ Marital status } \\
\hline Single & 140 & 19.0 \\
\hline Married (Monogamous) & 350 & 47.4 \\
\hline Married (Polygamous) & 146 & 19.8 \\
\hline Separated/Divorced & 75 & 10.1 \\
\hline Widowed & 27 & 3.7 \\
\hline \multicolumn{3}{|l|}{ Parity } \\
\hline $0-1$ & 208 & 28.2 \\
\hline $2-4$ & 243 & 32.9 \\
\hline $5-7$ & 149 & 20.2 \\
\hline Above 7 & 138 & 18.7 \\
\hline \multicolumn{3}{|l|}{ Educational attainment } \\
\hline No formal education & 24 & 3.3 \\
\hline Primary & 336 & 45.5 \\
\hline Secondary & 259 & 35.1 \\
\hline Tertiary & 119 & 16.1 \\
\hline \multicolumn{3}{|l|}{ Occupation } \\
\hline Housewife/Student & 65 & 8.8 \\
\hline Trading & 185 & 25.1 \\
\hline Farming & 252 & 34.1 \\
\hline Self employed & 96 & 13.0 \\
\hline Civil/public servant & 140 & 19.0 \\
\hline \multicolumn{3}{|l|}{ Monthly income } \\
\hline Low $(0$ - 50,000$)$ & 418 & 56.6 \\
\hline $\begin{array}{l}\text { Middle ( } \$ 51,000- \\
\# 100,000)\end{array}$ & 190 & 25.8 \\
\hline $\begin{array}{l}\text { High ( } \$ 101,000 \text { and } \\
\text { Above) }\end{array}$ & 130 & 17.6 \\
\hline
\end{tabular}

Table 1: Socio-demographic characteristics of the respondents

(51.5\%) or due to financial constraints (21.4\%). Other reasons given include belief/culture (14.4\%), Wait for the father to come back (7\%) and distance (5.7\%).

\section{Relationship between time of seeking malaria treatment for children and Socio-demographic characteristics of mothers}

Statistical relationships between time of seeking treatment for children with malaria and socio-demographic characteristics of mothers is depicted in Table 2 . The result $(\chi 2 \mathrm{cal} .=55.34$, d.f $=12, \mathrm{P}$ $=0.05$, as against $\mathrm{x} 2 \mathrm{tab} .=21.0261)$ showed that there was a significant relationship between age of mothers and delay in seeking treatment for malaria for under-five children. There was also a significant relationship between marital status of mothers and time of seeking treatment for malaria for under-five children $(\chi 2 \mathrm{cal} .=162.4$, d.f $=12, \mathrm{P}=0.05$ as against $\mathrm{x} 2$ tab.21.0261). Furthermore, a significant association was found between malaria treatment time for children (0-5 years) and parity (number of childbirth) of mothers $(\chi 2 \mathrm{cal} .=315.3$, d.f $=9, \mathrm{p}=$ 0.05 and $\mathrm{x} 2 \mathrm{tab} .=16.9190)$.

\section{Relationship between time of seeking malaria treatment for children and Socio- economic characteristics of mothers}

Statistical relationships between time of seeking treatment for children with malaria and socio-economic characteristics of mothers is shown in Table 3. Educational attainment $(\chi 2$ cal. $=355-918, \mathrm{df}=12$,
$\mathrm{P}=0.05)$ as well as occupation of mothers $(\chi 2$ cal. $=312.55, \mathrm{df}=12$, $P=0.05)$ showed very significant relationships with time for seeking malaria for under-five children. The results further showed a significant relationship between family monthly income and time taken to seek malaria treatment for children $(0-5$ years $)(\chi 2 \mathrm{cal} .=562.9, \mathrm{df}=6, \mathrm{P}=$ $0.05)$.

\section{Discussion}

The study unraveled the independent variables associated delay is seeking timely and appropriate treatment for childhood ( 0 - 5 years) malaria in Ohaji/Egbema Local Government Area, which are rural communities of South - Eastern Nigeria.

In this study, it was discovered that, out of Seven Hundred and Thirty-Eight mothers sampled, only $22 \%$ of them sought treatment for their children perceived as having malaria within 24 hours of noticing the first sign/symptom. The remaining $78 \%$ did not seek treatment within 24 hours, that is, they delayed in seeking malaria treatment for their children $(0-5$ years). This is in contrast to a report by Ahorlu et al. [16], in a similar study in Southern Ghana. According to them, only $11 \%$ of the respondents sought treatment within 24 hours. This disparity could be attributed to increased awareness and improved attitude of the people toward programmes and initiatives aimed at eradicating malaria. Difference in socio-cultural practices and beliefs between the two countries (Nigeria and Ghana) could as well be a contributory factor.

On their reasons for delay, most of the mothers (51.5\%) claimed they had to watch the child for some days because they wanted to ascertain the cause of the illness but in line with the report of Muela [17].

Regarding choice of first line treatment the mothers who did not delay were $32 \%$ and $28.2 \%$ likely to patronize the health centre and clinics/hospitals respectively. Almost half of the mothers $(36.3 \%)$ who delayed treated their children at home with drugs that are no longer effective against malaria in Nigeria. Their next option was patronizing Patent Medicine dealers, instead of seeking early and appropriate treatment from health centres, clinics, hospitals or even using recommended drug at home $[18,19]$. Also, some of the mothers (19.7\%) used local herbs as their first line choice for malaria treatment in their children. This is in accordance with the report of Okeke et al. [11].

The commonest reason that affected their choices of firstline treatment was cost $30.5 \%$ which was followed by belief/culture 28.5 . This finding contrasts with report of a similar study in Orissa Disrict, India - where distance (66.9\%) was the commonest reason giving by the majority of the mothers [20].

The statistical analysis of data obtained in this study showed that the younger mothers had to wait for the decision of their husbands (resulting to delay) before seeking treatment for malaria in under-five children. This is in conformity with the report of Cowan in a similar study in Northern Ghana [21].

It was discovered that mothers in monogamous marriages were more likely to seek treatment for their under-five children late than mothers in polygamous marriages. This is because mothers in polygamous marriages had to compete with each other in everything including child rearing. The mothers in polygamous marriages believed that the one with more children attract more attention, love and blessings from their husband. This finding is in line with the report 
Citation: Chukwuocha UM, Okpanma AC, Chukwuocha AN, Nwakwuo GC (2015) Social Determinants of Malaria Treatment Seeking Time by Mothers for Children (0-5 Years) in South Eastern Nigeria. J Trop Dis 2: 154. doi: 10.4172/2329891X.1000154

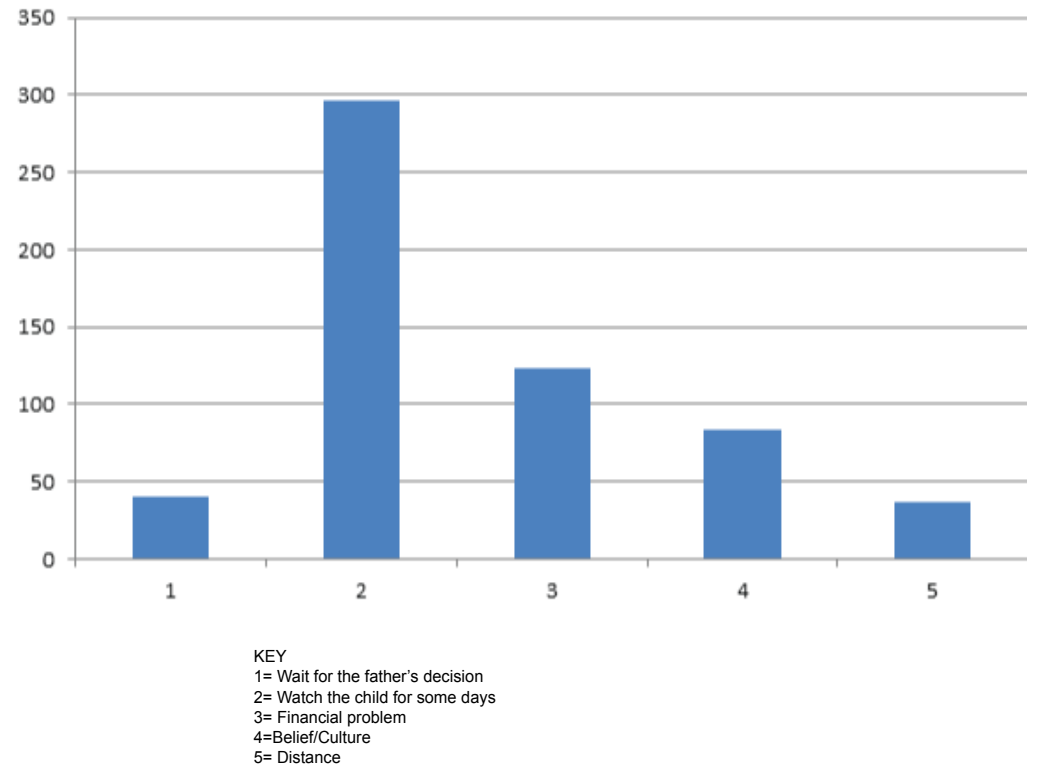

Figure 1: Reasons for not seeking malaria treatment within 24 hours

\begin{tabular}{|c|c|c|c|c|c|c|}
\hline \multirow[t]{2}{*}{ Variables } & \multicolumn{4}{|c|}{ Time of Seeking treatment (hours) } & \multirow[t]{2}{*}{$x^{2}$} & \multirow[t]{2}{*}{ p-value } \\
\hline & Within 24hrs & $25-48$ & $49-72$ & 73 \& above & & \\
\hline Age of mother & & & & & 55.34 & $<0.05$ \\
\hline $15-21 y r s$ & $8(19.4)$ & $12(22.7)$ & $40(26.5)$ & $28(9.4)$ & & \\
\hline $22-28 y r s$ & $48(53.4)$ & $59(62.3)$ & $72(72.8)$ & $63(53.4)$ & & \\
\hline $29-35 y r s$ & $42(48.8)$ & $72(56.9)$ & $65(66.5)$ & $42(48.8)$ & & \\
\hline $36-42 y r s$ & $53(36.9)$ & $43(22.6)$ & $42(50.2)$ & $29(36.9)$ & & \\
\hline $43-49 y r s$ & $12(4.4)$ & $4(5.1)$ & $3(6.0)$ & $1(4.4)$ & & \\
\hline Marital status & & & & & 162.4 & $<0.05$ \\
\hline Single & $32(30.9)$ & $70(45.3)$ & $20(44.8)$ & $18(19)$ & & \\
\hline $\begin{array}{c}\text { Married } \\
\text { (Monogamous) }\end{array}$ & $28(77.3)$ & $100(113.3)$ & 164(111.9) & $58(47.4)$ & & \\
\hline Married (Polygamous) & $48(32.3)$ & $42(47.3)$ & $38(46.7)$ & $18(19.8)$ & & \\
\hline Separated/Divorced & $38(16.6)$ & $21(24.3)$ & $10(24)$ & $6(10.2)$ & & \\
\hline Widowed & $17(6.0)$ & $6(8.7)$ & $4(8.7)$ & $0(3.7)$ & & \\
\hline Parity & & & & & 315.3 & $<0.05$ \\
\hline $0-1$ & $118(45.9)$ & $42(47.9)$ & $28(59.8)$ & $20(54.4)$ & & \\
\hline $2-4$ & $22(53.7)$ & $92(56.0)$ & $70(69.8)$ & $59(63.5)$ & & \\
\hline $5-7$ & $15(32.9)$ & $22(34.3)$ & $74(42.8)$ & $38(39.0)$ & & \\
\hline Above 7 & $8(30.5)$ & $14(31.8)$ & $40(39.6)$ & $76(36.1)$ & & \\
\hline
\end{tabular}

Table 2: Relationship between Sociodemographic factors and time of seeking malaria Treatment for children (0-5years)

[22]. They reported that children of monogamous parents were three times more likely to have delay in diagnosis and treatment of malaria when compared to children who had polygamous parents. Also, the mothers from monogamous marriages had to wait for the decision of their husbands before taking action when their children contract malaria. This finding is in agreement with that of Kamat [23].

The occupational status of the mother was found to be strongly associated with delay in seeking malaria treatment for children (0-5 years). This is occasioned by lack of women empowerment in decision making as some of the women said they have to wait for the 
Citation: Chukwuocha UM, Okpanma AC, Chukwuocha AN, Nwakwuo GC (2015) Social Determinants of Malaria Treatment Seeking Time by Mothers for Children (0-5 Years) in South Eastern Nigeria. J Trop Dis 2: 154. doi: 10.4172/2329891X.1000154

Page 5 of 6

\begin{tabular}{|c|c|c|c|c|c|c|}
\hline \multirow[t]{2}{*}{ Variables } & \multicolumn{4}{|c|}{ Time of Seeking treatment (hours) } & \multirow[t]{2}{*}{$x^{2}$} & \multirow[t]{2}{*}{ p-value } \\
\hline & Within 24hrs & $25-48$ & $49-72$ & $73 \&$ above & & \\
\hline $\begin{array}{l}\text { Educational } \\
\text { attainment }\end{array}$ & & & & & 335.918 & $<0.05$ \\
\hline No formal education & $3(5.3)$ & $6(6.3)$ & $13(3.8)$ & $2(6.6)$ & & \\
\hline Primary & $22(74.2)$ & $56(88.8)$ & $92(80.6)$ & $166(92.4)$ & & \\
\hline Secondary & $58(57.2)$ & $96(68.4)$ & $70(86.1)$ & $35(71.2)$ & & \\
\hline Tertiary & $80(26.3)$ & $37(31.4)$ & $2(28.5)$ & $0(32.7)$ & & \\
\hline Occupation & & & & & 312.55 & $<0.05$ \\
\hline Housewife/Student & $12(14.4)$ & $38(15)$ & $8(18.7)$ & $7(17)$ & & \\
\hline Trading & 11(40.9) & $46(42.6)$ & 78(53.1) & $50(48.4)$ & & \\
\hline Farming & $16(55.7)$ & $26(22.1)$ & $44(27.6)$ & $22(25.1)$ & & \\
\hline Self employed & $4(21.2)$ & $26(22.1)$ & $44(27.6)$ & 114(65.9) & & \\
\hline Civil/public servant & $120(30.9)$ & 18(32.2) & $2(40.2)$ & $0(36.6)$ & & \\
\hline Monthly income & & & & & 562.9 & $<0.05$ \\
\hline Low $(0-\$ 50,000)$ & $2(92.3)$ & $28(65.1)$ & $172(24.6)$ & $216(135.9)$ & & \\
\hline $\begin{array}{l}\text { Middle ( } \$ 1,000 \text { - } \\
\$ 100,000)\end{array}$ & $53(42.0)$ & $75(29.6)$ & $40(56.6)$ & $22(61.8)$ & & \\
\hline $\begin{array}{l}\text { High ( } \$ 101,000 \text { and } \\
\text { Above) }\end{array}$ & $108(28.7)$ & $12(20.3)$ & $8(38.8)$ & $2(42.3)$ & & \\
\hline
\end{tabular}

Table 3: Relationship between Socioeconomic factors and time of seeking malaria Treatment for children (0-5years)

father's decision. This finding is in line with the report from Malawi by Chibwana et al., in a similar study in Mwanza-Neno District of Malawi [24]. They reported that women are not empowered to make decisions affecting positive health seeking behaviour independent of their husbands or other family members. The finding from this study also conforms to report from Tanzania [25]. Although, this contrasts with the report from South West Ethiopia [22], this difference could be as a result of difference in geographical characteristics and sociocultural practices and perceptions of the two countries (Nigeria and Ethiopia). The educational attainment of the mothers was also found to be associated with delay in seeking malaria treatment for their children (0-5 years). As the educational attainment increases, the delay in seeking treatment for childhood (0-5 years) becomes minimal. It conforms to the report of a similar study in Tanzania [25]. But it contrasts with the report from South West Ethiopia [22]. This could be blamed on the level of awareness on causes, treatment, prevention and complications of malaria in the areas of study.

The result of the statistical analysis of data in this study showed a significant relationship between socio-economic status of the family and delay in malaria treatment for under-five children. The families in low socio-economic class were likely to delay than those in the middle and higher classes. This supports the findings of other previous studies [26-28].

The study revealed that mothers with more number of children tend to have lackadaisical attitude towards their children when they develop signs/symptoms of malaria. They preferred home based treatment with left over drugs from previous ailment or patronize patent medicine dealers. This finding conforms to the report of a similar study in North eastern Nigeriaby Kwabe et al. [29]. According to them, the proportion of nomadic Fulani mothers who managed fever at home was higher among the group with parity of $6-7$ (52.6\%) when compared with other lower parity groups. The parity of the mothers showed significant relationship with delay in malaria treatment when analyzed using the fisher's exact (15.987, $\mathrm{p}$-value $=0.002)$ as well as in chi-square test of independence $(\mathrm{X} 2=315.3, \mathrm{df}=9$, $\mathrm{p}$-value $=0.05)$.
Also, mothers who gave history of child death tend to seek malaria treatment for their children (0-5) years earlier than those without such history. This has been reported by Getahun et al. [22].

\section{Conclusion}

Less than a quarter (22\%) of the mothers in Ohaji/Egbema local government Area of Imo state, Nigeria seek early and appropriate malaria treatment for children ( $0-5$ years) suspected of having malaria. This means that more than $2 / 3$ of the mothers $(78 \%)$ seek malaria treatment for their children ( $0-5$ years) late.

It was discovered that the $78 \%$ of the respondents who delayed in seeking malaria treatment made several wrong choices as first-line malaria treatment. These choices included treating with herbs, use of non-effective drugs (e.g. chloroquine etc.), patent medicine dealers and even prayer houses. The mothers who delayed gave several reasons which included waiting for the fathers' decision, watching the child for some days, financial problems, belief/culture and distance. The study revealed several determinants of malaria seeking delay which included marital status/type of marriage, age and occupational status of the mothers including their educational attainment, parity and history of child mortality. Other determinants were belief/culture, distance of residence from health facility and socio-economic status of the family. These results will therefore be very useful in formulation proper health education for women of child bearing age in rural endemic areas. Such women should also be helped to break some social barriers that restrain them from taking prompt and effective action for their ill children especially those with malaria.

\section{References}

1. Gupta P, Ghai OP (2007) Textbook of preventive and social medicine. New Delhi. Columbia Broadcasting System (CBS) Publishers.

2. Mayo Clinic (2013) Diseases and Conditions Malaria

3. Chan M (2011) Malaria Deaths are down but progress remains fragile. Geneva World Health Organization. King behaviour for febrile under-five children in Mwanzo-Neno District, Malawi. Malaria Journal 8: 219.

4. Centre for Disease Control (2001) Global malaria prevention and contro 
Citation: Chukwuocha UM, Okpanma AC, Chukwuocha AN, Nwakwuo GC (2015) Social Determinants of Malaria Treatment Seeking Time by Mothers for Children (0-5 Years) in South Eastern Nigeria. J Trop Dis 2: 154. doi: 10.4172/2329891X.1000154

Page 6 of 6

programme: Moving Ahead in the $21^{\text {st }}$ century.

5. United States Embassy in Nigeria (2012) Nigeria malaria fact sheet. Economic Sections.

6. Centre for Disease Control (2012a) Impact of malaria: Malaria worldwide. 24.7.

7. Centre for disease control (2012b) President Malaria Initiatives: Efforts to combat malaria. Atlanta. United States Agency for International Development/ Nigeria

8. Korenromp EL, William BG, Gouws F, Dye C RW (2003) Measurement of trends in childhood malaria mortality in Africa: An assessment of progress towards target based on verbal autopsy. Lancet Infect Dis. 3: 349- 358.

9. Chukwu COO (2011) Implementation Guide for parasite-based diagnosis of malaria. National Malaria Control Programme. Federal Ministry of Health, Abuja Nigeria.

10. World Health Organization (2013) Malaria Fact Sheet No: 94. WHO Media Centre. Geneva.

11. Okeke TA, Uzuchukwu, Okafor HU (2006) Improving childhood malaria treatment and referral practices by training patent medicine vendors in rural South-East Nigeria. Malaria Journal 8: 260.

12. Shaikh BT (2008) Understanding social determinants of health seeking behaviour providing a rational framework for health policy and systems development. J Pak Med Assoc. 58: 33-36

13. Safer MA, Tharps QJ, Jackson TC, Leventhal H (1979) Determinants of three stages of delay in seeking care at a medical clinic. Med Care. 17: 11-29

14. World Health Organization (1995) A draft proposal: A rapid assessment of health seeking behavior in relation to malaria. World Health Organization, Geneva.

15. Heggenhougen $\mathrm{KH}$, Hackethal V, Vivek $\mathrm{P}$ (2003) The behavioural and Social aspects of malaria and its control: An introduction and Annotated Bibliography. United Nations Development Programme/World Bank/World Health Organization Special programme for Research and training in Tropical Diseases 03.01.

16. Ahorlu CK, Koram KA, Ahorlu C, Savigny D, Weiss MG (2006) Socio-cultural determinants of treatment delay for childhood malaria in Southern Ghana. Trop Med Int Health. 11: 1022-1031.
17. Muela SH (2000) Community understanding of malaria and treatment seeking behaviour in a holoendemic area of Southern Tanzania. Inaugural Dissertation. University of Basel: 31-40.

8. Oberlander L, Elverdan B (2000) Malaria in the united republic of Tanzania: Cultural considerations and health seeking behavior. Bull World Health Organ 78: $1352-1357$.

19. Uzochukwu, Onyejekwe OE (2004) Socio-economic differences and healthseeking behavior for the diagnosis and treatment of malaria: A case study of four local government areas operating the Bamako Initiative Programme in South-East Nigeria. Malaria Journal 4: 13

20. Das A, Ravindran, T.K.S (2010) Factors affecting treatment-seeking for febrile Illness in a malaria endemic block in Boudha district, Orisa, India: policy Implication for Malaria Control. Malaria Journal 9: 377.

21. Cowan S (2007) Inequality in household decision-making for early treatment of malaria in young children: A case study in northern Ghana. GUJHS 4

22. Getahun A, Deribe K, Deribew A (2010) Determinants of delay in malaria Treatment-seeking behavior for under-five children in South-West Ethiopia: A Case control study. Malaria Journal 9: 320

23. Kamat V.R (2006) Cultural knowledge and micro politics of therapy seeking for childhood febrile illness in Tanzania. Soc Sci Med 62: 2945-2959.

24. Chibwana Cl, Mathanga DP, Chinkhumba J, Campbell CH (2009) Sociocultural preditors of health-se

25. Sekule $P$ (2007) Late health seeking behavior among care-takers of under-five children with malaria at Muhimbili National Hospital, Dar-es-saalam, Tanzania. Umea International School of public Health 1: 12-16

26. Jowett M, Miller N (2000). Malaria expenditure analysis: Tanzania case study. The University of York.

27. Jones CO, Williams HA (2004) The social burden of malaria: What are we measuring. American Journal of Tropical Medicine and Hygiene. 72: 156-161.

28. Agu A.P, Nwojiji JO (2005) Childhood malaria: Mothers' perception and treatment seeking behaviour in a community in Ebonyi State South-East Nigeria. Journal of Community Medicine \& Primary Healthcare 17: 45-50.

29. Kwabe TS, Abdulfatai O, Agbam EF, Usman A, Bashiru M (2013). Mothers management of malaria fever among under-five nomadic Fulani children of North-eastern Nigeria. AJIDM 1: 26-33 